\title{
Transmastoid reshaping of the sigmoid sinus: preliminary study of a novel surgical method to quiet pulsatile tinnitus of an unrecognized vascular origin
}

\author{
${ }^{*}$ Chong Sun Kim, MD, PhD, ${ }^{1,2}$ So Young Kim, MD, ${ }^{3}$ Hyunseok Choi, MD, ${ }^{3}$ Ja-Won Koo, MD, PhD, ${ }^{3}$ \\ Shin-Young Yoo, MD, PhD, ${ }^{4}$ Gwang Seok An, BS, ${ }^{5}$ Kyogu Lee, PhD, ${ }^{5}$ Inyong Choi, PhD, ${ }^{6}$ and \\ Jae-Jin Song, MD, PhD 3
}

\begin{abstract}
${ }^{1}$ Seoul National University College of Medicine; ${ }^{2}$ Soree Ear Clinic; ${ }^{4}$ Myungdong Yonsei ENT Clinic; ${ }^{5}$ Graduate School of Convergence Science and Technology, Seoul National University, Seoul; ${ }^{3}$ Department of Otorhinolaryngology-Head and Neck Surgery, Seoul National University Bundang Hospital, Seongnam, Korea; and ${ }^{6}$ Department of Communication Sciences and Disorders, The University of lowa, lowa City, lowa
\end{abstract}

\begin{abstract}
OBJECTIVE A dominant sigmoid sinus with focal dehiscence or thinning (DSSD/T) of the overlying bony wall is a commonly encountered, but frequently overlooked, cause of vascular pulsatile tinnitus (VPT). Also, the pathophysiological mechanism of sound perception in patients with VPT remains poorly understood. In the present study, a novel surgical method, termed transmastoid SS-reshaping surgery, was introduced to ameliorate VPT in patients with DSSD/T. The authors reviewed a case series, analyzed the surgical outcomes, and suggested the pathophysiological mechanism of sound perception. The theoretical background underlying VPT improvement after transmastoid SS-reshaping surgery was also explored.
\end{abstract}

METHODS Eight patients with VPT that was considered attributable to DSSD/T underwent transmastoid SS-reshaping surgery between February 2010 and February 2015. The mean postoperative follow-up period was 9.5 months (range 4-13 months). Transmastoid SS-reshaping surgery featured simple mastoidectomy, partial compression of the SS using harvested cortical bone chips, and reinforcement of the bony SS wall with bone cement. Perioperative medical records, imaging results, and audiological findings were comprehensively reviewed.

RESULTS In 7 of the 8 patients (87.5\%), the VPT abated immediately after surgery. Statistically significant improvements in tinnitus loudness and distress were evident on numeric rating scales. Three patients with preoperative ipsilesional low-frequency hearing loss exhibited postoperative improvements in their low-frequency hearing thresholds. No major postoperative complications were encountered except in the first subject, who experienced increased intracranial pressure postoperatively. This subsided after a revision operation for partial decompression of the SS.

CONCLUSIONS Transmastoid SS-reshaping surgery may be a good surgical option in patients with DSSD/T, a previously unrecognized cause of VPT. Redistribution of severely asymmetrical blood flow, reinforcement of the bony SS wall with bone cement to reconstruct a soundproof barrier, and disconnection of a problematic sound conduction route via simple mastoidectomy silence VPT.

http://thejns.org/doi/abs/10.3171/2015.6.JNS15961

KEY WORDS pulsatile tinnitus; cranial sinuses; mastoid; surgical procedures, skull base

$\mathrm{T}$ INNITUS can be subjective, i.e., when the sound is perceived by the individual alone, or objective, i.e., when an observer can recognize the tinnitus sound of the patient. ${ }^{2}$ Subjective tinnitus comes from the inner ear, ascending auditory pathway, or auditory/nonauditory cortical regions. ${ }^{9,22-24,26-28,30}$ The origin of objective tinnitus is vascular or nonvascular. Of them, vascular objective tinnitus is also known as pulsatile tinnitus (PT), featuring perception of a pulse-synchronous or rhythmical sound. ${ }^{2}$

Although PT constitutes less than $10 \%$ of all tinnitus, ${ }^{10,21}$

ABBREVIATIONS CTA = CT angiography; DSSD/T = dominant sigmoid sinus with dehiscence or thinning; IICP = increased intracranial pressure; IJV = internal jugular vein; MRI/A = MRI with angiography; NRS = numeric rating scale; $\mathrm{PT}=$ pulsatile tinnitus; $\mathrm{PTA}=$ pure-tone audiometry; $\mathrm{SS}=$ sigmoid sinus; $\mathrm{SSD}=\mathrm{SS}$ dehiscence; TB = temporal bone; VPT = vascular PT.

SUBMITTED April 27, 2015. ACCEPTED June 18, 2015.

INCLUDE WHEN CITING Published online January 8, 2016; DOI: 10.3171/2015.6.JNS15961.

* Drs. C. S. Kim and S. Y. Kim contributed equally to this work. 
accurate diagnosis of PT is imperative because 44\%-91\% of PT patients have underlying structural abnormalities treatable via surgical or endovascular intervention. ${ }^{10,11} \mathrm{PT}$ cases amenable to treatment include those of arterial or venous origin, or those associated with neoplastic problems triggering intracranial pulsation, vascular flow murmur, temporomandibular joint problems, patulous eustachian tube, myoclonus of the palate or middle ear muscle, or a dehiscent semicircular canal. ${ }^{12}$

Vascular PT (VPT), the most common form of PT, is attributable to turbulent blood flow caused by vascular abnormalities including an aberrant internal carotid artery, a dehiscent jugular bulb, a vascular loop in the internal auditory canal, an arteriovenous fistula, atherosclerosis, and sigmoid sinus (SS) dehiscence or diverticulum. ${ }^{1}$ SS dehiscence (SSD) and diverticula are the most common identifiable causes of VPT. SSD or diverticula are evident in $23 \%$ of patients with PT, and, among PT patients with definable etiologies, $33 \%$ had SSD or diverticula. ${ }^{7,20}$ However, SSD is also found in as many as $12 \%$ of asymptomatic patients, ${ }^{7}$ and a bone thickness of less than $0.6 \mathrm{~mm}$ over the SS can be falsely interpreted as dehiscence. ${ }^{3,14}$ Also, when it is considered that iatrogenic dehiscence of the bony wall of the SS during mastoidectomy usually does not trigger PT, SSD alone may not always cause VPT. Rather, other anatomical abnormalities associated with conduction of blood flow-driven sound to the middle ear may be associated with VPT in patients with true- or false-positive SSD.

Recently, we reported a case of objective unilateral PT audible via bare-ear auscultation. ${ }^{31}$ Temporal bone CT angiography (TB-CTA) revealed an ipsilaterally prominent SS (the dominance of which was stark compared with that of the contralateral SS). However, the affected SS exhibited no dehiscence or diverticulum. We successfully managed the case via transmastoid compression of the dominant SS using 2 chips of autologous cortical bone; the subjective loudness of the PT was alleviated to $10 \%$ of the preoperative level. Based on this initial experience, we sought to identify patients with unilateral PT who had a significant ipsilateral-dominant SS with dehiscence or thinning (DSSD/T). Of such patients, a subgroup with incapacitating symptoms underwent surgical interventions.

Below, we introduce our novel modified surgical technique with transmastoid reshaping of the DSSD/T. We used simple mastoidectomy, mechanical compression of the SS, and reinforcement of the bony SS wall for management of VPT caused by DSSD/T. We discuss the surgical outcomes. Additionally, we define the possible pathophysiological mechanism of VPT perception and show that symptoms improve after surgical intervention in certain patients.

\section{Methods \\ Patient Population}

We included patients with PT who visited Seoul National University Bundang Hospital between February 2010 and February 2015. After radiological evaluation via contrast-enhanced TB-CTA and brain MRI with angiography (MRI/A), patients for whom the PT was presumed to be caused only by DSSD/T were selected as candidates for transmastoid SS-reshaping surgery. Patients with PT that was probably caused by other structural abnormalities evident on TB-CTA or brain MRI/A were not scheduled for SS reshaping. A total of 8 patients underwent transmastoid SS-reshaping surgery. Patient demographic characteristics are summarized in Table 1. Patients' ages ranged from 36 to 66 years (median 56 years). Of the 8 patients, 3 were men and 5 were women. Four patients complained of right-sided PT, and the other 4 had leftsided PT. Upon TB-CTA with contrast enhancement, all patients exhibited DSSD/T on the side of the perceived PT. The study was approved by the institutional review board of the Clinical Research Institute at Seoul National University Bundang Hospital.

\section{Preoperative Clinical Evaluation}

All patients in the current case series underwent comprehensive preoperative evaluation in terms of the side of the tinnitus; its duration; other otologic symptoms such as hearing loss or dizziness; any medical condition that might be associated with PT such as previous cerebrocranial trauma, cardiovascular disorders, metabolic syndrome, or psychiatric disorders; and the patient's medication history, including angiotensin-converting enzyme inhibitors and calcium antagonists. Also, all patients underwent numeric rating scale (NRS) analysis of tinnitus loudness (i.e., by answering the question, "How loud is your tinnitus?" on a scale from 0 to 10) and tinnitus-related distress (i.e., by answering the question, "How much are you bothered by your tinnitus?," again on a scale from 0 to 10 ) by a physician who was not involved in the surgical procedures.

All patients underwent full otoendoscopic and audiometric evaluations, including pure-tone audiometry (PTA) and psychoacoustic tinnitus pitch and loudness matching. On physical examination, the head and neck area was fully examined, including the changes of PT upon digital compression of the ipsilateral internal jugular vein (IJV) and head rotation to the ipsi- and contralateral sides, and auscultation to locate any possible source of PT. In selected patients (Cases 4 and 7 in Table 1), the PT acoustical characteristics were analyzed via transcanal tinnitus sound recording using an inserted microphone (RØDE Microphones); real-time recording using Cubase 5.0 software (Steinberg Media Technologies); and, finally, data analysis using MATLAB R2013a (The MathWorks).

All patients underwent TB-CTA with contrast enhancement using Philips128 CT scanners (Philips Medical Systems). TB-CTA was acquired with a tube energy of $120 \mathrm{kVp}$, a quality reference value of $250 \mathrm{~mA}$, and a detector configuration of $2 \times 0.625 \mathrm{~mm}$. The thickness or interval of each CT scan was $0.7 \mathrm{~mm}$ and the length of each scan was $16 \mathrm{~cm}$. SSD on TB-CTA was defined as loss of the normal SS contour, focal thinning of the mastoid cortex, absence of normal cortical bone over the SS, or direct contact of the SS with mastoid air cells. ${ }^{5}$

\section{Surgical Technique}

In all cases, the surgical goals were as follows: 1) to fully skeletonize the dominant SS and to reshape that SS via external compression; 2) to reinforce the SS wall 
TABLE 1. Summary of clinical and demographic data in 8 patients with PT

\begin{tabular}{|c|c|c|c|c|c|c|c|c|c|}
\hline $\begin{array}{l}\text { Case } \\
\text { No. }\end{array}$ & $\begin{array}{l}\text { Age } \\
\text { (yrs) }\end{array}$ & Sex & $\begin{array}{l}\text { Medical } \\
\text { Illness }\end{array}$ & $\begin{array}{c}\text { Tinnitus } \\
\text { Duration (mos) }\end{array}$ & $\begin{array}{l}\text { Side } \\
\text { of PT }\end{array}$ & Imaging \& Intraop Dx & $\begin{array}{l}\text { Postop Change } \\
\text { of PT }\end{array}$ & $\begin{array}{l}\text { Postop Follow- } \\
\text { Up (mos) }\end{array}$ & $\begin{array}{c}\text { Postop } \\
\text { Complications }\end{array}$ \\
\hline 1 & 58 & M & None & 24 & $\mathrm{Lt}$ & Dominant SS w/o any bony dehiscence & Relieved & 13 & $\begin{array}{l}\text { IICP leading to } \\
\text { reexploration }\end{array}$ \\
\hline 2 & 66 & $\mathrm{~F}$ & ESRD & 3 & Rt & $\begin{array}{l}\text { Dominant SS w/ dehiscence at the } \\
\text { transverse sinus-SS junction }\end{array}$ & Cured & 10 & None \\
\hline 3 & 53 & $\mathrm{~F}$ & None & 12 & $\mathrm{Lt}$ & Dominant SS w/o any bony dehiscence & Stationary & 10 & None \\
\hline 4 & 36 & $\mathrm{~F}$ & None & 6 & Lt & $\begin{array}{l}\text { Dominant SS w/ dehiscence at the } \\
\text { transverse sinus-SS junction }\end{array}$ & Relieved & 9 & None \\
\hline 5 & 62 & M & None & 3 & Rt & $\begin{array}{l}\text { Dominant SS w/ dehiscence at the } \\
\text { transverse sinus-SS junction }\end{array}$ & Cured & 7 & None \\
\hline 6 & 41 & $\mathrm{~F}$ & None & 3 & Rt & $\begin{array}{l}\text { Dominant SS w/ dehiscence at the } \\
\text { transverse sinus-SS junction }\end{array}$ & Cured & 6 & None \\
\hline 7 & 39 & $\mathrm{~F}$ & None & 3 & Rt & Dominant SS w/o any bony dehiscence & Relieved & 4 & None \\
\hline 8 & 54 & M & None & 12 & Lt & Dominant SS w/o any bony dehiscence & Cured & 4 & None \\
\hline
\end{tabular}

$\mathrm{Dx}=$ diagnosis; $\mathrm{ESRD}=$ end-stage renal disease.

(including any dehiscent portion), to reduce transmission of pulsatile sound from the SS; and 3) to perform complete simple mastoidectomy to reduce sound conduction from the SS to the middle ear. The surgical steps of our transmastoid SS reshaping are summarized in Fig. 1. Via a postauricular incision, an anteriorly based periosteal flap was elevated and the mastoid cortex widely exposed. Two or 3 autologous cortical bone chips were harvested for later use (Fig. 1A). Via simple mastoidectomy, the SS was fully skeletonized (Fig. 1B). After evaluating dehiscence or thinning of the skeletonized SS wall, the transverse sinus-SS junction was exposed by uncovering the overlying bony shell (Fig. 1C, dotted ellipse).

Next, a piece of harvested temporalis fascia was inserted between the SS and the skeletonized shell (Fig. 1D, dotted ellipse), and the harvested autologous cortical bone chips were inserted between the fascia and the SS wall, parallel to the long axis of the SS, to compress the SS externally (Fig. 1E, dotted ellipse). In this step, to smoothen the transverse sinus-SS junction without causing postoperative central venous hypertension, we compressed less than $30 \%$ of the preoperative SS diameter. After covering the bony defect with another piece of temporalis fascia, the skeletonized SS wall was fully reinforced by covering it with HydroSet Injectable HA Bone Substitute (Stryker Corp.) (Fig. 1F). Surgery was completed by reapproximating the periosteal and skin flaps.

\section{Postoperative Management and Outcome Measurements}

To prevent the postoperative development of increased intracranial pressure (IICP), as in Case 1 of our series,,$^{31}$ all subsequent patients were routinely prescribed amiloride $5 \mathrm{mg}$ and hydrochlorothiazide $25 \mathrm{mg}$ twice daily for 2 weeks postoperatively. On each follow-up visit to the outpatient clinic, NRS tinnitus intensity and NRS tinnitusrelated distress were measured by a physician who was not involved in the surgical procedures. PTA was performed to detect any noticeable change in hearing threshold. Also, follow-up TB-CTA with contrast enhancement was performed postoperatively in all patients.

\section{Statistical Analysis}

The significance of improvements in NRS tinnitus loudness and NRS tinnitus-related distress was determined using the Wilcoxon signed-rank test. Statistical analyses were performed using SPSS (version 20.0; SPSS, Inc.). Statistical significance was set at $\mathrm{p}<0.05$.

\section{Results}

\section{Preoperative Findings}

The median NRS tinnitus loudness and NRS tinnitus-
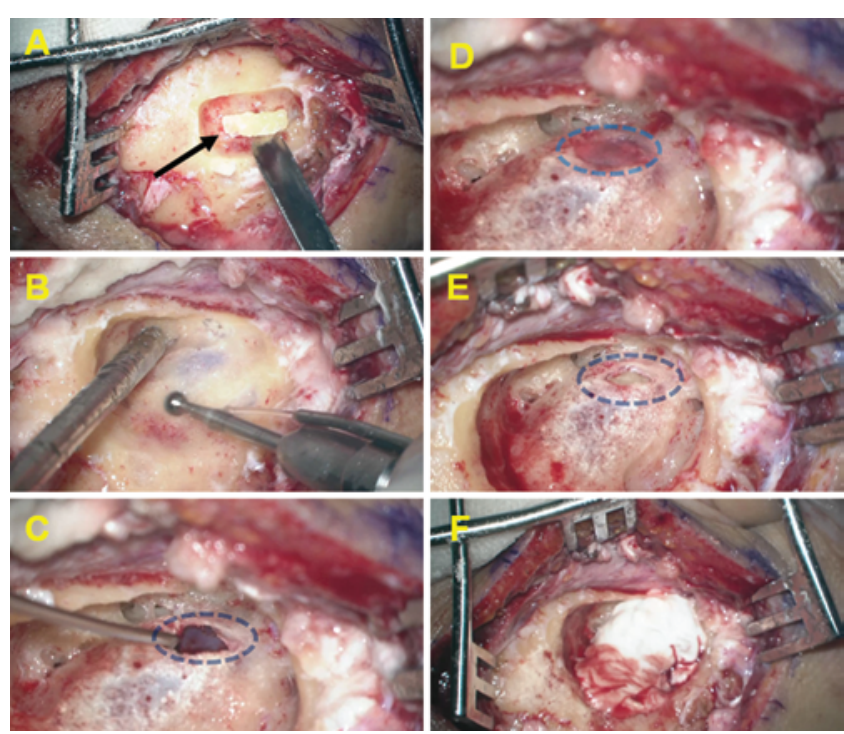

FIG. 1. Photographs showing the procedures of SS-reshaping surgery. Harvesting of autologous cortical bone chips from the mastoid cortex (A); complete simple mastoidectomy and skeletonization of the SS (B); exposure of the transverse sinus-SS junction by uncovering the overlying bony shell (dotted ellipse) (C); coverage of the exposed area of the SS with harvested temporalis fascia (dotted ellipse) (D); external compression of the SS with the autologous cortical bone chips (dotted ellipse) (E); application of bone cement to reinforce the bony SS wall (F). Figure is available in color online only. 
A Case 1, preoperative

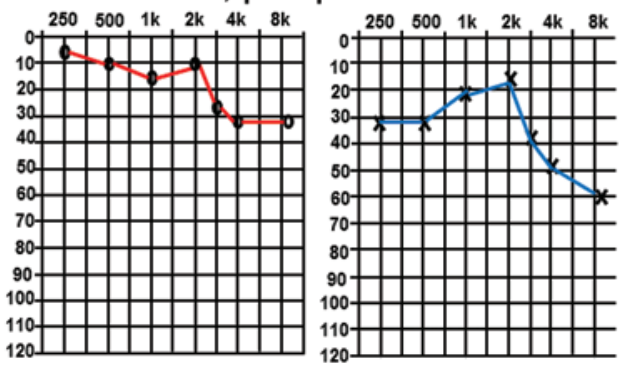

C Case 3, preoperative

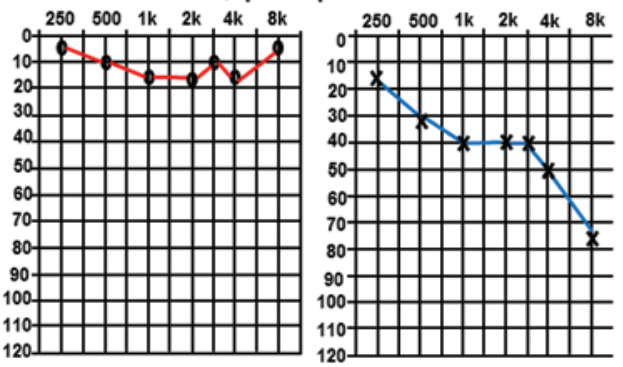

\section{E Case 5, preoperative}
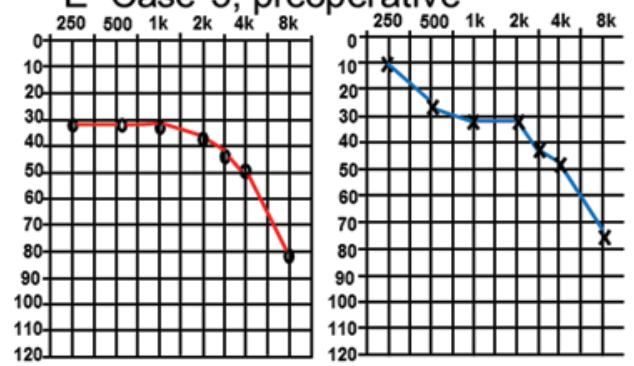

B Case 1, postoperative

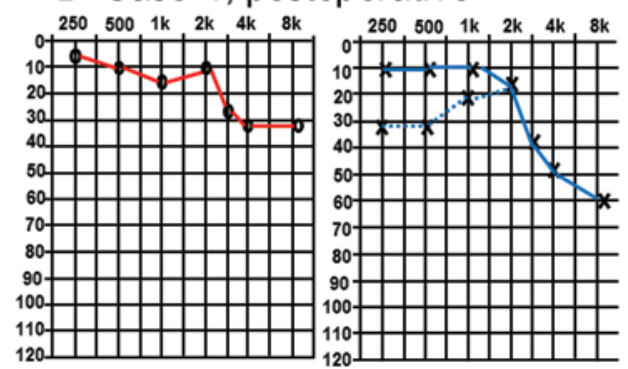

D Case 3, postoperative

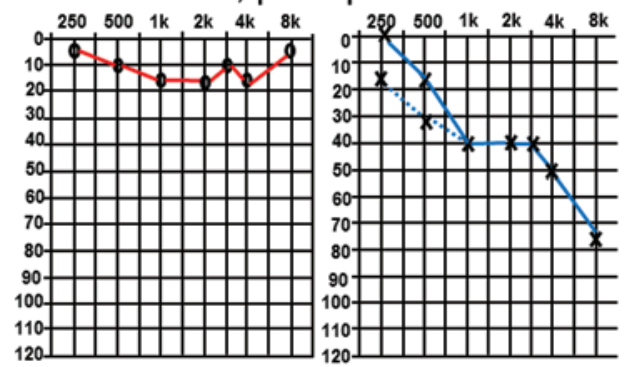

F Case 5, postoperative

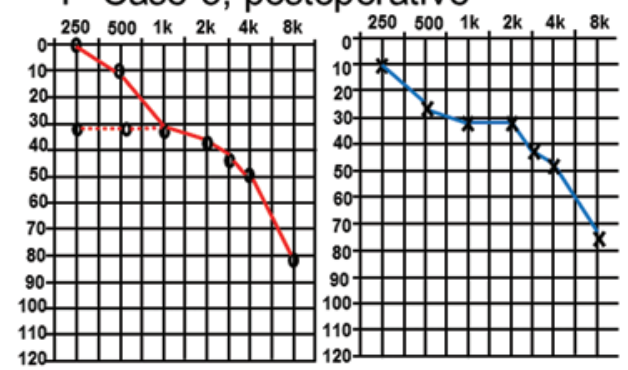

FIG. 2. Comparison of pre- and postoperative PTAs. Three patients (Cases 1, 3, and 5) exhibited ipsilesional low-frequency sensorineural hearing loss in preoperative audiograms (A, C, and E); improvements in the low-frequency hearing thresholds were evident postoperatively (B, D, and F). Figure is available in color online only.

related distress scores were 6 (range 3-9) and 7.5 (range $2-10)$, respectively. The median duration of PT was 5 months (range 3-24 months) (Table 1). One patient (Case 2) suffered from end-stage renal disease. Otherwise, there were no patients with a medical illness that may have affected their PT.

No patients complained of other otologic symptoms, including dizziness or ear fullness. Upon PTA, 3 patients (Cases 1, 3, and 5 in Table 1) exhibited mild ipsilesional low-frequency sensorineural hearing loss (the PTA thresholds were more than $30 \mathrm{~dB}$ at $0.25 \mathrm{kHz}$ or $0.5 \mathrm{kHz}$ ) but normal contralateral low-frequency thresholds (Fig. 2A, $\mathrm{C}$, and $\mathrm{E}$; left panels). There were no cases of conductive component of hearing loss with air-bone gaps on PTA. Otoendoscopic or other physical examination of the head and neck area revealed no abnormal finding in any subject. All patients reported decreased sound intensity upon digital compression of the ipsilateral IJV or head rotation to the ipsilateral side, whereas increased sound intensity was reported upon head rotation to the contralateral side.

Upon bare-ear transcanal auscultation with a stethoscope or a Toynbee tube, PT was audible in only 1 patient (Case 1). Transcanal tinnitus sound recording was per- formed in Cases 4 and 7; both exhibited pulse-synchronous signals. Time-domain analysis revealed that pulsesynchronous signals were augmented upon contralateral head rotation and diminished upon ipsilateral rotation. This is because head rotation to the ipsilateral side decreases venous blood flow due to ipsilateral IJV compression by contraction of the sternocleidomastoid muscle, whereas rotation to the contralateral side increases venous blood flow ${ }^{8,10}$ (Fig. 3, upper panels). Spectral-temporal analysis revealed audible low-frequency signals (Fig. 3, lower panels).

On TB-CTA, all subjects except Case 3 exhibited absolute ipsilesional dominance of the SS, and 7 of 8 patients (87.5\%) had suspicious ipsilesional dehiscence/thinning. However, Case 3 exhibited relatively low-level dominance of the ipsilesional SS. Dehiscence or thinning of the bony SS wall was mostly centered at the transverse sinus-SS junction. All patients exhibited bilaterally well-pneumatized mastoid cavities (Fig. 4, left panels).

\section{Intraoperative Findings}

As described above, all subjects underwent simple mastoidectomy and skeletonization of the SS, and expo- 

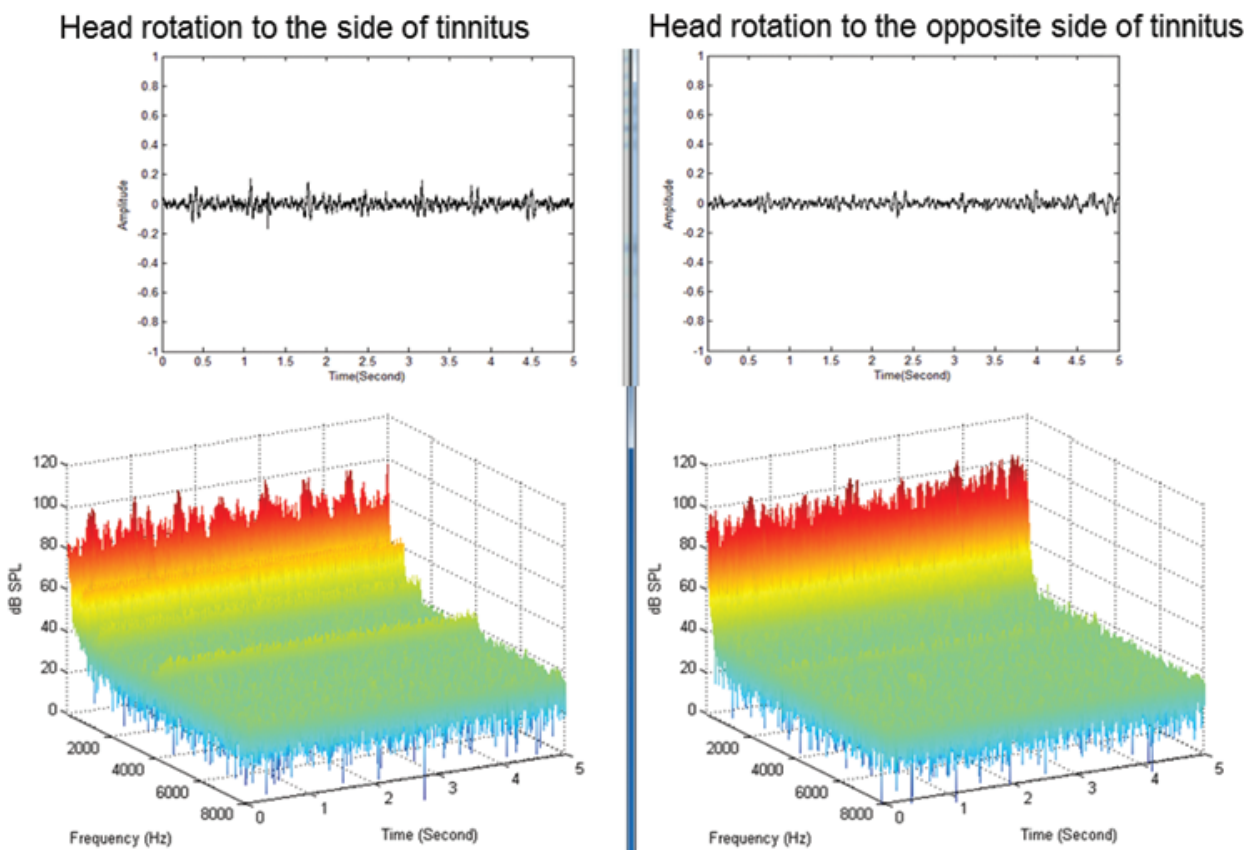

FIG. 3. Spectral-temporal analysis of signals obtained via transcanal sound recording. Pulse-synchronous sound signals were detected on time-domain analysis (upper panels). Signals were augmented upon contralateral head rotation (left panels) and diminished upon ipsilateral head rotation (right panels). The spectral-temporal analysis revealed audible low-frequency signals (lower panels). Figure is available in color online only.

sure of the thinned or dehiscent bony wall. Next, the SS was reshaped by external compression of the sinus and reinforcement of the bony wall with bone cement. Intraoperatively, 4 of 8 subjects exhibited true bony dehiscence at the junction between the transverse sinus and the SS, whereas the other 4 exhibited focal thinning of the SS wall, without any dehiscence. No surgery featured a major intraoperative event.

\section{Postoperative Findings}

In 7 of the 8 patients $(87.5 \%)$, the PT abated immediately after the operation. Of note, in 4 of these 7 patients, the PT resolved completely. No patient reported changes in the residual tinnitus or other symptoms (e.g., headache) during position change, exertion, or other activities. However, in Case 3, the patient complained of persistent symptoms that did not improve during 10 months of follow-up. The median postoperative follow-up period was 9.5 months (range 4-13 months). The median postoperative NRS tinnitus intensity and NRS tinnitus-related distress scores were 1 (range $0-10$ ) and 1 (range $0-10$ ), respectively (Fig. 5). The improvements in NRS tinnitus loudness and NRS tinnitus-related distress were statistically significant $(\mathrm{p}=$ 0.007 and $p=0.008$, respectively, determined using the Wilcoxon signed-rank test). None of the patients showed changes in their NRS tinnitus intensity and NRS tinnitusrelated distress scores during the postoperative follow-up described in Table 1.

Interestingly, all 3 patients who exhibited preoperative ipsilesional low-frequency sensorineural hearing loss exhibited improvements in low-frequency hearing thresholds immediately after operation (Fig. 2B, D, and
F; right panels). Postoperative TB-CTA revealed reshaped SSs, with smaller diameters and thicker bony walls, in all cases (Fig. 4, right panels). As mentioned above, postoperative IICP developed in Case 1. The patient complained

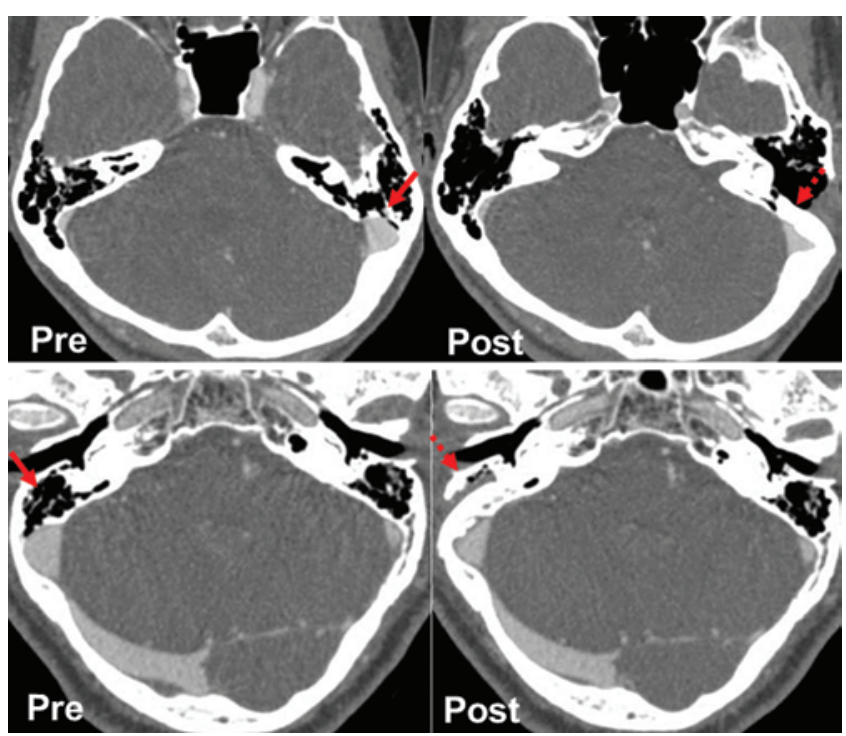

FIG. 4. Comparison of pre- and postoperative TB CT data with contrast enhancement. A suspicious dehiscence at the bony SS wall and an ipsilesional-dominant SS (upper left panel, solid arrow) are reshaped by external compression of the SS and reinforcement of the bony SS wall with bone cement (upper right panel, dotted arrow). Cells in the pneumatic mastoid area and the DSSD/T (lower left panel, solid arrow) are reshaped via transmastoid SS-reshaping surgery (lower right panel, dotted arrow). Figure is available in color online only. 

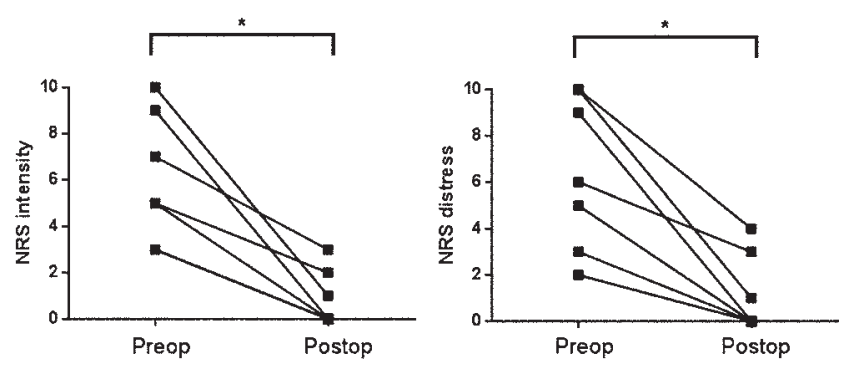

FIG. 5. Changes in NRS values of tinnitus loudness and tinnitus-related distress are plotted. All patients exhibited statistically significant improvements in NRS tinnitus loudness and NRS tinnitus-related distress scores immediately after operation. Asterisks indicate statistically significant changes. Postoperative $=$ postoperative scores; preoperative $=$ preoperative scores.

of pulsating headache, and papilledema was noted on the funduscopy. After confirming near obstruction of the SS on MR venography of the brain, surgical reexploration and partial decompression of the SS were performed. Partial removal of the inserted pieces of cortical bone chips completely relieved his headache and resumed the venous flow of the SS, as confirmed by follow-up MR venography immediately after reoperation. However, none of the other 7 cases developed any major postoperative complications after routine short-term prescription of diuretics.

\section{Discussion}

Seven of 8 patients $(87.5 \%)$ reported markedly decreased or absent VPT immediately after transmastoid SS-reshaping surgery; this was statistically significant $(\mathrm{p}$ $=0.001$ in terms of NRS tinnitus loudness and $p=0.004$ in terms of NRS tinnitus-related distress, determined using the Wilcoxon signed-rank test). The results cannot be directly compared with previous studies because this is the first case series to examine patients with VPT due to DSSD/T. However, the outcome is comparable to those of previously reported transmastoid SS surgical interventions in patients with VPT due to SS diverticulum and/or dehiscence, which demonstrated successful outcomes in all enrolled case series. ${ }^{5,16,19}$ Moreover, 3 cases exhibited post- operative low-frequency hearing gains (Fig. 2). These improvements can be attributed to alleviation of the masking effect of VPT on (characteristically) low-pitched sound..$^{21}$

Pulsatile tinnitus can originate from vascular structures of the intracranial, extracranial, cervical, and thoracic cavities. ${ }^{21}$ The evaluation of medical histories, physical examination, and imaging studies such as CT and/or MRI/A are important for differentiating the vascular origin of VPT (Table 2), ${ }^{1,2,8}$ The possible etiologies of PT were previously explored, ${ }^{8,21,29}$ but DSSD/T has never been considered a possible cause of VPT. After we noted the postoperative improvement in VPT symptoms in our first case (an ipsilesionally prominent SS lacking any dehiscence or a diverticulum), we searched for previously unrecognized DSSD/T in patients with VPT who otherwise exhibited no abnormal vascular structural finding on either TB-CTA or MRI/A. As a result, we successfully managed a further 7 patients with VPT using our novel surgical approach. Below, we discuss the possible pathophysiological mechanism underlying VPT generation and perception, and the advantages of our surgical procedure.

\section{Possible Pathophysiological Mechanisms of VPT Perception in Patients With DSSD/T}

Blood flow generates a constant pulse-synchronous sound that is not usually consciously perceived. However, in VPT cases of venous origin, 2 plausible mechanisms may cause patients to perceive the sound. First, local turbulence originating from acceleration or changes in blood flow may disrupt laminar flow and become the source of VPT. ${ }^{8}$ Second, as previous studies have suggested,,$^{19,20}$ when a pulse-synchronous sound is generated, that sound may be transmitted primarily through the bone conduction route to the inner ear if any discontinuity or weakness is present in the bony (normally) soundproof wall.

Vascular PT caused by DSSD/T can be explained by the mechanisms summarized above. Unilateral SS dominance is a common finding, and the right venous system is generally dominant. ${ }^{15}$ However, when the asymmetry between the 2 SSs is abnormally severe, the abnormally dominant side may receive almost all of the venous blood flow, considering that the volume flow rate is proportional to the fourth power of the radius (Poiseuille's law) (Fig. 6A, left

TABLE 2. Possible causes and clinical examinations for PT

\begin{tabular}{|c|c|c|c|}
\hline \multirow[b]{2}{*}{ Location } & \multirow[b]{2}{*}{ Causes } & \multicolumn{2}{|c|}{ Examinations } \\
\hline & & Common & Specific \\
\hline Arterial & $\begin{array}{l}\text { Stenosis, aneurysms, atherosclerotic carotid artery disease, tortuous } \\
\text { internal carotid artery, carotid artery dissection, increased cardiac } \\
\text { output (anemia, thrombocytosis, thyrotoxicosis, \& pregnancy) }\end{array}$ & $\begin{array}{l}\text { Medical histories such as cere- } \\
\text { brocranial trauma, medica- } \\
\text { tion, hyperthyroidism }\end{array}$ & $\begin{array}{l}\text { Strong arterial compres- } \\
\text { sion of the internal } \\
\text { carotid artery }\end{array}$ \\
\hline $\begin{array}{l}\text { Arteriovenous } \\
\text { transition }\end{array}$ & $\begin{array}{l}\text { Dural arteriovenous fistulas, direct arteriovenous fistulas, arteriove- } \\
\text { nous malformations, vessel-rich tumors, capillary hyperemia }\end{array}$ & & \\
\hline Venous & $\begin{array}{l}\text { Intracranial hypertension, jugular bulb abnormalities (high location, } \\
\text { dehiscence, \& diverticula), transverse-SS stenosis \& aneurysms, } \\
\text { abnormal condylar \& mastoid emissary veins, IICP associated w/ } \\
\text { Arnold-Chiari syndrome, \& stenosis of the sylvian aqueduct }\end{array}$ & $\begin{array}{l}\text { Blood pressure, body mass } \\
\text { index, testing for anemia, } \\
\text { neurological examinations }\end{array}$ & $\begin{array}{l}\text { Slight venous compres- } \\
\text { sion, Valsalva maneu- } \\
\text { ver, Müller maneuver, } \\
\text { rotation of the head }\end{array}$ \\
\hline Nonvascular & Semicircular canal dehiscence, other & $\begin{array}{l}\text { Imaging studies: CT/CTA, MR/ } \\
\text { MRA }\end{array}$ & $\begin{array}{l}\text { Audiometry, vestibular } \\
\text { function test }\end{array}$ \\
\hline
\end{tabular}




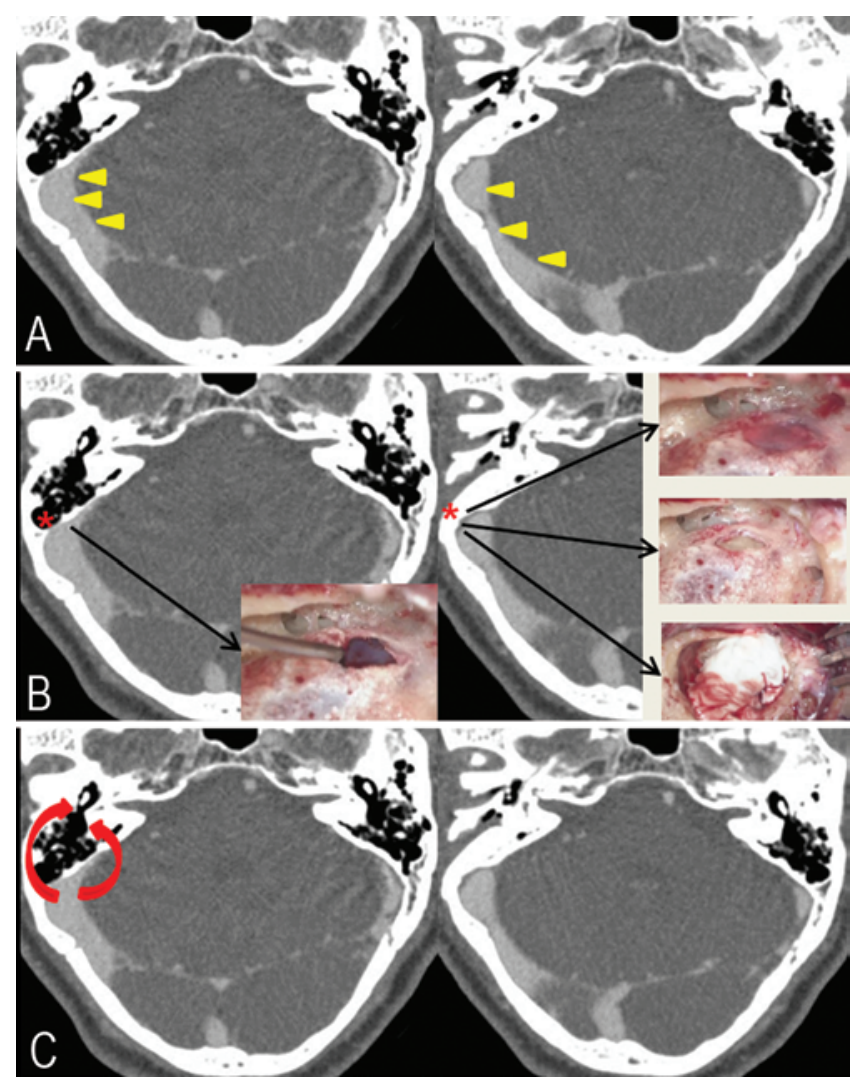

FIG. 6. The surgical procedures of transmastoid SS-reshaping surgery are displayed. A: First, the ipsilesional-dominant SS causing severely asymmetrical blood flow (left panel, arrowheads) is compressed using cortical bone chips (to redistribute the blood flow; right panel, arrowheads). B: Next, the dehiscent or thinned bony wall of the SS (causing sound leakage; left panel, asterisk) is reinforced with HydroSet Injectable HA Bone Substitute (right panel, asterisk). C: Also, the pneumatic mastoid cavity that allows sound conduction from the SS to the middle ear (left panel, curved arrows) is disconnected via simple mastoidectomy (right panel). Figure is available in color online only.

panel). ${ }^{18}$ This may trigger slowly progressive erosion of the cortical bone overlying the significantly dominant SS and, as the result of a form of Lamarckian inheritance, the diameter of the dominant SS may increase gradually (Fig. $6 \mathrm{~A}$, left panel). There have been no studies that directly explored the longitudinal changes in the diameter of the SS. However, a recent study on the results of SS diverticulum repair has shown recurrence of the diverticulum 4.5 years after initial resurfacing surgery using only bone wax..$^{25}$ This report may indirectly prove that a significantly dominant SS may result in the erosion of the overlying cortical bone and thus increase its own diameter.

As suggested previously, ${ }^{16}$ acute pressure erosion of or congenital defects in the SS bony wall are unlikely; the $\mathrm{SS}$ is a low-pressure venous system. Also, in most cases reported to date, PT symptoms caused by venous abnormalities have developed in late middle age.,16 This is probably why our youngest patient with PT was in her mid-30s.

Increased irregularity of or asymmetry in vessel diameter, thus not the absolute vessel diameter per se, is considered to generate the turbulence causing VPT. ${ }^{4}$ It has been suggested that the shape and angle of the transverse sinusSS junction are important in the generation of turbulent flow that causes resonant sound in the mastoid cavity. ${ }^{32}$ In agreement with previous reports, most dehiscence or focal thinning of the bony SS wall was evident at the transverse sinus-SS junction in our case series.

A poor soundproof interface between the SS and the middle ear may reduce dampening of VPT sound (Fig. 6B, left panel). Thus, pulse-synchronous VPT can be transmitted to the middle ear via air or bone conduction. ${ }^{19}$ Because all of our subjects had well-pneumatized mastoid air cells, the VPT may have been transmitted from a dehiscent or thinned SS wall to the middle and inner ear through the trabecular structure of such air cells, thus via both air and bone conduction routes, with minimal sound dampening (Fig. 6C, left panel).

\section{The Advantages of Transmastoid SS-Reshaping Surgery Compared With Other External and Endovascular Approaches}

After treating our first patient (Case 1), who exhibited marked improvement of VPT symptoms upon external compression of the SS using cortical bone chips, and mild recurrence after partial removal of the bone chips to control IICP, we refined our transmastoid SS-reshaping surgery; this is now our routine procedure for selected patients with VPT who have ipsilesional DSSD/T.

As summarized in Figs. 6 and 7, the surgical rationale for transmastoid SS-reshaping surgery is 3-fold. First, by inserting cortical bone chips between the SS and its bony wall, partial redistribution of the blood flow to minimize the turbulence may be achieved (Fig. 6A, right panel; Fig. 7). In particular, by smoothing the transverse sinusSS junction using harvested cortical bone chips, the most plausible source of turbulence is interrupted. Second, to reestablish the sound-insulating effect of the bony SS wall, reinforcement of that wall, not only at the dehiscent or thinned portion but along its entire length, is achieved using bone cement (Fig. 6B, right panel; Fig. 7). Third, simple mastoidectomy removes mastoid air cells to fully expose the SS from the transverse sinus-SS junction down to the jugular bulb, and to minimize bone conduction from the SS to the middle ear (Fig. 6C, right panel; Fig. 7).

An SS-resurfacing procedure including external compression of the diverticulum and resurfacing of the bony wall (via a transmastoid approach) to manage VPT patients with SS diverticula has been described. ${ }^{33}$ This procedure is similar to our SS-reshaping surgery, but may not be appropriate for VPT patients with DSSD/T. Unlike what is useful for patients with SS diverticula, focal reinforcement of the dehiscent/thinned portion may not adequately reduce local turbulence in VPT patients with DSSD/T. In particular, as in Cases 1, 7, and 8 in our series, patients lacking any dehiscence of the bony SS wall may be encountered. In such cases, a simple SS-resurfacing procedure is not useful.

An endovascular approach has been successfully used to treat VPT patients with ipsilateral SS diverticula. ${ }^{6,13,17}$ Endovascular occlusion of the sinus diverticulum may be a good (and less invasive) alternative to surgery if the anatomy of the venous lesion allows coils to be success- 


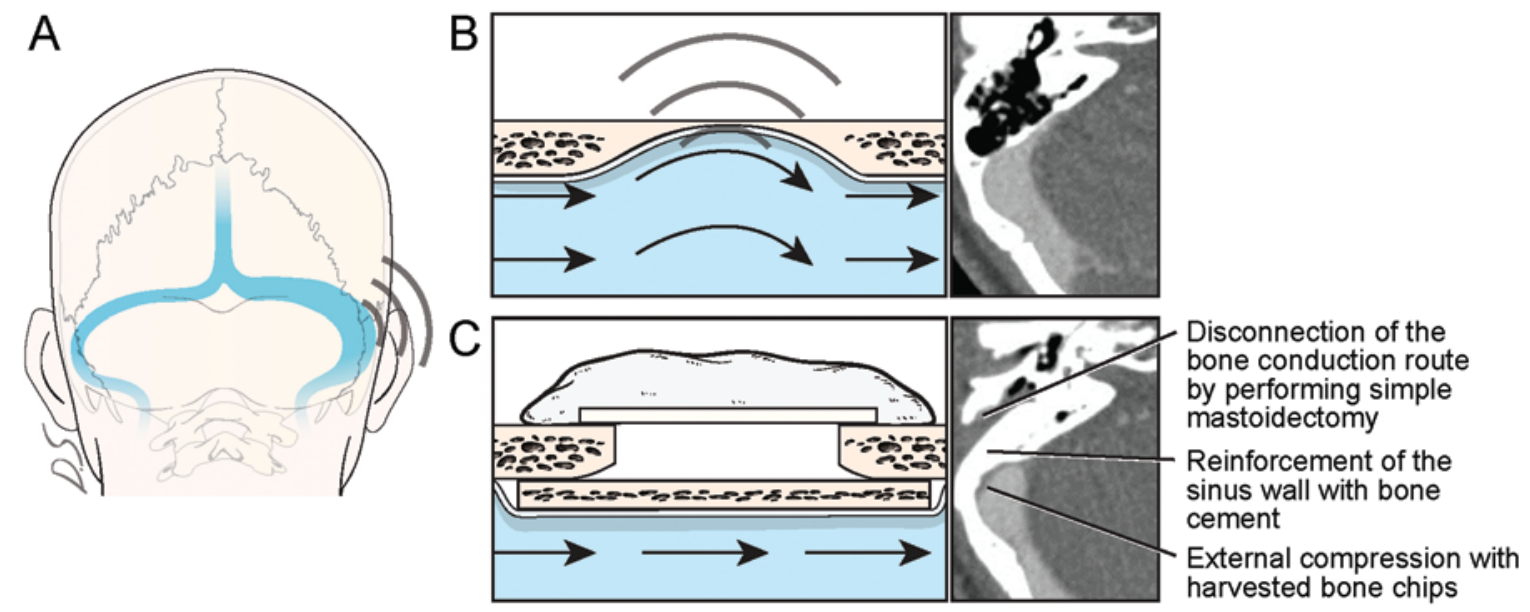

FIG. 7. Schematic summary of the surgical procedures of transmastoid SS-reshaping surgery. The ipsilesional-dominant SS causing severely asymmetrical blood flow (A) may generate pulse-synchronous sound, which is conducted via dehiscent or thinned bony wall (B). A combination of external compression of the SS with harvested cortical bone chips, reinforcement of the SS wall, and complete simple mastoidectomy quiets PT (C). Figure is available in color online only

fully maintained. However, as in our case series, if VPT is generated not by a focal vascular lesion but rather by a $\mathrm{DSSD} / \mathrm{T}$, the endovascular approach may not be as effective as noted in patients with SS diverticula. Therefore, SSreshaping surgery is better than an endovascular approach to treat subjects such as those in our current case series, affording more reliable control of sound generation.

\section{Selection of Surgical Candidates}

After achieving excellent results in Cases 1 and 2, the symptoms of Case 3 did not improve after surgery. Although the audiogram revealed an improvement in the ipsilesional low-frequency threshold (Fig. 2D), which may indicate that the masking effect of VPT had been reduced, the patient's symptoms were persistent. This may be attributable to a relatively nonsignificant dominance of the ipsilesional SS compared with the other 7 subjects in our current case series. To solve the problem, we used transcanal VPT sound recording in the preoperative assessment of 2 patients (Cases 4 and 7; in Cases 5 and 6, the recording was not possible due to technical issues). Transcanal VPT recording/spectral-temporal analysis is not specific for the identification of turbulence at the level of the transverse sinus-SS junction because it only suggests that the presumptive origins are located at or near the middle ear. However, by confirming the presence of VPT and the possible sound source at or near the middle ear by this objective measurement and combining spectral-temporal analysis results with imaging findings of DSSD/T, we could select surgical candidates.

\section{Limitations of the Present Study}

To the best of our knowledge, this is the first report on surgical reshaping for VPT patients with DSSD/T. However, our case series was limited in several aspects. The number of subjects was small and the follow-up was short; we could not evaluate the durability of reshaped sinus walls or redistribution of blood flow, nor the long-term surgical outcomes. Further clinical experience with a larger patient group is required to optimize the surgical technique and to establish criteria for optimal candidate selection. In addition, we only indirectly assessed symptom improvement using NRS scales because we cannot yet objectively compare pre- and postoperative symptoms. Therefore, it is possible that the surgical outcomes were confounded by a placebo effect of performing the surgical procedure itself. To minimize this possibility, the NRS scales were repeatedly measured at each visit to the outpatient clinic by a third party. In this regard, further work on objective measurements of PT is warranted. Also, VPT due to DSSD/T might be abated only by performing cortical mastoidectomy and sinus decompression without SS reshaping and resurfacing, especially in patients with an SS diverticulum closer to the jugular bulb. This warrants future randomized controlled trials to determine the optimal procedure.

\section{Conclusions}

Transmastoid SS-reshaping surgery efficiently controls VPT in subjects with DSSD/T, a previously unrecognized cause of VPT. Appropriate surgical candidates may be selected via meticulous preoperative radiological evaluation; psychoacoustic measurements are also warranted. A combination of external compression of the SS with harvested cortical bone chips, reinforcement of the SS wall, and complete simple mastoidectomy allowed $87.5 \%$ of patients in our current case series to experience immediate resolution of or significant improvement in symptoms.

\section{Acknowledgments}

This work was supported by a grant from the Korea Health Technology R\&D Project through the Korea Health Industry Development Institute (KHIDI), funded by the Ministry of Health \& Welfare, Republic of Korea (grant number HI14C2264).

\section{References}

1. Ahsan SF, Seidman M, Yaremchuk K: What is the best imag- 
ing modality in evaluating patients with unilateral pulsatile tinnitus? Laryngoscope 125:284-285, 2015

2. Baguley D, McFerran D, Hall D: Tinnitus. Lancet 382:16001607,2013

3. Carey JP, Minor LB, Nager GT: Dehiscence or thinning of bone overlying the superior semicircular canal in a temporal bone survey. Arch Otolaryngol Head Neck Surg 126:137147,2000

4. Cho IK, Jung JY, Yoo DS, Suh MW: 3-dimensional reconstruction of the venous system in patients suffering from pulsatile tinnitus. Acta Otolaryngol 132:285-289, 2012

5. Eisenman DJ: Sinus wall reconstruction for sigmoid sinus diverticulum and dehiscence: a standardized surgical procedure for a range of radiographic findings. Otol Neurotol 32:1116-1119, 2011

6. Gard AP, Klopper HB, Thorell WE: Successful endovascular treatment of pulsatile tinnitus caused by a sigmoid sinus aneurysm. A case report and review of the literature. Interv Neuroradiol 15:425-428, 2009

7. Grewal AK, Kim HY, Comstock RH III, Berkowitz F, Kim HJ, Jay AK: Clinical presentation and imaging findings in patients with pulsatile tinnitus and sigmoid sinus diverticulum/dehiscence. Otol Neurotol 35:16-21, 2014

8. Hofmann E, Behr R, Neumann-Haefelin T, Schwager K: Pulsatile tinnitus: imaging and differential diagnosis. Dtsch Arztebl Int 110:451-458, 2013

9. Langguth B, Kreuzer PM, Kleinjung T, De Ridder D: Tinnitus: causes and clinical management. Lancet Neurol 12:920-930, 2013

10. Liyanage SH, Singh A, Savundra P, Kalan A: Pulsatile tinnitus. J Laryngol Otol 120:93-97, 2006

11. Madani G, Connor SE: Imaging in pulsatile tinnitus. Clin Radiol 64:319-328, 2009

12. Mattox DE, Hudgins P: Algorithm for evaluation of pulsatile tinnitus. Acta Otolaryngol 128:427-431, 2008

13. Mehanna R, Shaltoni H, Morsi H, Mawad M: Endovascular treatment of sigmoid sinus aneurysm presenting as devastating pulsatile tinnitus. A case report and review of literature. Interv Neuroradiol 16:451-454, 2010

14. Mondina M, Bonnard D, Barreau X, Darrouzet V, FrancoVidal V: Anatomo-radiological study of the superior semicircular canal dehiscence of 37 cadaver temporal bones. Surg Radiol Anat 35:55-59, 2013

15. Ohata K, Haque M, Morino M, Nagai K, Nishio A, Nishijima Y, et al: Occlusion of the sigmoid sinus after surgery via the presigmoidal-transpetrosal approach. J Neurosurg 89:575584, 1998

16. Otto KJ, Hudgins PA, Abdelkafy W, Mattox DE: Sigmoid sinus diverticulum: a new surgical approach to the correction of pulsatile tinnitus. Otol Neurotol 28:48-53, 2007

17. Park YH, Kwon HJ: Awake embolization of sigmoid sinus diverticulum causing pulsatile tinnitus: simultaneous confirmative diagnosis and treatment. Interv Neuroradiol 17:376379, 2011

18. Roos A: Poiseuille's law and its limitations in vascular systems. Med Thorac 19:224-238, 1962

19. Santa Maria PL: Sigmoid sinus dehiscence resurfacing as treatment for pulsatile tinnitus. J Laryngol Otol 127 (Suppl 2):S57-S59, 2013

20. Schoeff S, Nicholas B, Mukherjee S, Kesser BW: Imaging prevalence of sigmoid sinus dehiscence among patients with and without pulsatile tinnitus. Otolaryngol Head Neck Surg 150:841-846, 2014

21. Sismanis A: Pulsatile tinnitus: contemporary assessment and management. Curr Opin Otolaryngol Head Neck Surg 19:348-357, 2011

22. Song JJ, De Ridder D, Schlee W, Van de Heyning P, Vanneste S: "Distressed aging": the differences in brain activity between early- and late-onset tinnitus. Neurobiol Aging 34:1853-1863, 2013

23. Song JJ, De Ridder D, Van de Heyning P, Vanneste S: Mapping tinnitus-related brain activation: an activation-likelihood estimation metaanalysis of PET studies. J Nucl Med 53:1550-1557, 2012

24. Song JJ, De Ridder D, Weisz N, Schlee W, Van de Heyning P, Vanneste S: Hyperacusis-associated pathological resting-state brain oscillations in the tinnitus brain: a hyperresponsiveness network with paradoxically inactive auditory cortex. Brain Struct Funct 219:1113-1128, 2014

25. Song JJ, Kim YJ, Kim SY, An YS, Kim K, Lee SY, et al: Sinus wall resurfacing for patients with temporal bone venous sinus diverticulum and ipsilateral pulsatile tinnitus. Neurosurgery 77:709-717, 2015

26. Song JJ, Punte AK, De Ridder D, Vanneste S, Van de Heyning P: Neural substrates predicting improvement of tinnitus after cochlear implantation in patients with single-sided deafness. Hear Res 299:1-9, 2013

27. Song JJ, Vanneste S, De Ridder D: Dysfunctional noise cancelling of the rostral anterior cingulate cortex in tinnitus patients. PLoS One 10:e0123538, 2015

28. Song JJ, Vanneste S, Schlee W, Van de Heyning P, De Ridder D: Onset-related differences in neural substrates of tinnitusrelated distress: the anterior cingulate cortex in late-onset tinnitus, and the frontal cortex in early-onset tinnitus. Brain Struct Funct 220:571-584, 2015

29. Sonmez G, Basekim CC, Ozturk E, Gungor A, Kizilkaya E: Imaging of pulsatile tinnitus: a review of 74 patients. Clin Imaging 31:102-108, 2007

30. Vanneste S, De Ridder D: The auditory and non-auditory brain areas involved in tinnitus. An emergent property of multiple parallel overlapping subnetworks. Front Syst Neurosci 6:31, 2012

31. Wee JH, Song JJ, Koo JW, Kim CS: Increased intracranial pressure after surgical treatment of pulsatile tinnitus from a prominent sigmoid sinus. Otol Neurotol 33:e41-e42, 2012

32. Xue J, Li T, Sun X, Liu Y: Focal defect of mastoid bone shell in the region of the transverse-sigmoid junction: a new cause of pulsatile tinnitus. J Laryngol Otol 126:409-413, 2012

33. Zhao H, Liu Y, Yang Y, Zeng X, Li J, Qiu S, et al: [2 cases of pulsatile tinnitus caused by sigmoid sinus diverticulum and literature review.] Lin Chung Er Bi Yan Hou Tou Jing Wai Ke Za Zhi 27:1088-1090, 2013

\section{Disclosures}

The authors report no conflict of interest concerning the materials or methods used in this study or the findings specified in this paper.

\section{Author Contributions}

Conception and design: Song. Acquisition of data: Song, An, Lee. Analysis and interpretation of data: Song, SY Kim, An, Lee, Choi. Drafting the article: SY Kim. Critically revising the article: all authors. Reviewed submitted version of manuscript: Song, CS Kim, SY Kim, Choi, Koo, Yoo. Approved the final version of the manuscript on behalf of all authors: Song. Statistical analysis: Song. Administrative/technical/material support: Song, CS Kim, Choi, Koo, Yoo.

\section{Correspondence}

Jae-Jin Song, Department of Otorhinolaryngology-Head and Neck Surgery, Seoul National University Bundang Hospital, 300 Gumi-dong, Bundang-gu, Seongnam 463-707, Republic of Korea. email: jjsong96@gmail.com. 